\title{
Gastrointestinal bleeding after left ventricular assist device implantation: It is all about the platelets
}

\author{
Juan A. Crestanello, MD
}

\footnotetext{
From the Division of Cardiac Surgery, Richard M. Ross Heart Hospital, The Ohio State University Wexner Medical Center, Columbus, Ohio.

Disclosures: Author has nothing to disclose with regard to commercial support.

Received for publication Sept 15, 2015; accepted for publication Sept 17, 2015; available ahead of print Oct 28, 2015.

Address for reprints: Juan A. Crestanello, MD, Division of Cardiac Surgery, The Ohio State University Wexner Medical Center, Richard M. Ross Heart Hospital, 410 W 10 Ave, Doan N 816, Columbus, OH 43210 (E-mail: juan.crestanello@osumc.edu).

J Thorac Cardiovasc Surg 2016;151:228-9

$0022-5223 / \$ 36.00$

Copyright (C) 2016 by The American Association for Thoracic Surgery

http://dx.doi.org/10.1016/j.jtcvs.2015.09.065
}

Continuous-flow (CF) left ventricular assist devices (LVADs) have revolutionized the management of patients with end-stage heart failure, increasing access and improving survival and quality of life. ${ }^{1}$ Notwithstanding their benefits, CF-LVADs are associated with multiple side effects. ${ }^{1}$ None of them is more puzzling than the increased incidence of bleeding, and of gastrointestinal bleeding (GI) in particular. Gastrointestinal bleeding is seen in approximately $20 \%$ of patients with CF-LVADs, with an annualized rate of 0.3 bleeding episodes/patient-y. ${ }^{2}$

The cause of the increased incidence of gastrointestinal bleeding in patients with CF-LVADs is multifactorial. Several interconnected factors have been postulated: (1) anticoagulation and antiplatelet therapy, (2) absence of pulsatile flow, (3) acquired von Willebrand factor (vWF) deficiency, (4) bowel angiodysplasias, and (5) platelet dysfunction.

In this issue of the Journal, Grosman-Rimon and colleagues ${ }^{3}$ have contributed to expand our knowledge of one of the least understood mechanisms of bleeding after CF-LVAD: platelet dysfunction. They have reported on 19 patients with CF-LVADs, $21 \%$ of whom had gastrointestinal bleeding. Serum cyclic guanosine monophosphate (cGMP) level was twice as high in patients with gastrointestinal bleeding as in patients without gastrointestinal bleeding. They also observed a progressive increase in serum levels of nitric oxide (NO) with the duration of LVAD support. Platelet-derived growth factor (PDGF) levels were lower in patients with LVAD support. Unfortunately, Grosman-Rimon and colleagues ${ }^{3}$ did not directly assess platelet function.

This study remind us of the potential mechanisms involved in the pathogenesis of gastrointestinal bleeding after CF-LVAD and of the complex interactions between them (as illustrated in the figure). It also provides an opportunity to review the central role of platelet dysfunction in the pathogenesis of gastrointestinal bleeding.

First, NO and cGMP are potent platelet inhibitors that play key roles in the regulation of platelet function. ${ }^{4}$

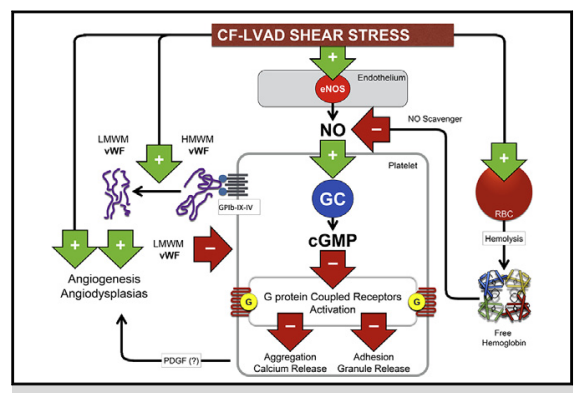

Diagram depicting central role of platelets in $\mathrm{Gl}$ bleeding after CF-LVAD implantation.

\section{Central Message}

Platelet dysfunction plays a central role in gastrointestinal bleeding after continuous-flow left ventricular assist devices.

See Article page 219.

See Editorials page 10 and 13.

Grosman-Rimon and colleagues ${ }^{3}$ noticed an increase in NO levels with the duration of support, which may be responsible for platelet inactivation. Although endothelial NO synthase is overexpressed both in heart failure and in patients with LVAD support, ${ }^{5}$ the net effect of CF-LVADs on NO levels is unclear because plasma free hemoglobin, a potent NO scavenger, is also increased as a result of hemolysis. NO activates platelet cytosolic guanylyl cyclase to generate cGMP, which blocks platelet activation, including calcium release, $\mathrm{G}$ protein activation, adhesion, granule release, and aggregation. ${ }^{4}$ cGMP blocks platelet activation by thrombin, thromboxane, collagen, vWF, and fibrinogen. ${ }^{4}$ The association of gastrointestinal bleeding with higher cGMP levels in the study of Grosman-Rimon and colleagues ${ }^{3}$ supports the role of cGMP-mediated platelet inhibition as a key component in the pathogenesis of gastrointestinal bleeding. In addition, the cyclic adenosine monophosphate-mediated platelet inhibitory pathway is also activated in patients with CF-LVAD support, as demonstrated by impairment in adenosine diphosphate-mediated platelet activation. $^{6}$

Second, CF-LVAD support is associated with acquired von Willebrand disease. ${ }^{7}$ Under normal circumstances, vWF exists as high-molecular weight multimers (HMWMs) that activate platelets by binding to platelet glycoprotein Ib-IX-IV receptor in presence of denuded 
endothelium and exposed collagen. ${ }^{6}$ vWF further affects platelet function by regulating guanyl cyclase activity and cGMP synthesis. CF-LVAD-induced shear stress leads to cleavage of HMWM vWF into low-molecular weight multimers (LMWMs). Plasma HMWM vWF and vWF activities are decreased in $70 \%$ of patients with CF-LVAD support, resulting in impaired vWF-dependent platelet aggregation. ${ }^{6}$ This impairment is more pronounced in patients with severe gastrointestinal bleeding. Shear stress also impairs platelet glycoprotein Ib-IX-V receptor function, which further increases bleeding. ${ }^{6}$ In acquired von Willebrand disease, LMWMs not only fail to activate platelets but may also directly inhibit them.

Third, CF-LVADs are associated with bowel mucosa angiodysplasias, which are the source of gastrointestinal bleeding in $31 \%$ of patients with CF-LVAD support. ${ }^{8}$ The lack of pulsatile flow, the associated changes in the bowel microcirculation, and the proangiogenic effects of LMWM vWF fragments are some of the mechanisms associated with the formation of angiodysplasias. Wever-Pinzon and colleagues ${ }^{9}$ demonstrated that reduced pulsatility index results in higher rates of gastrointestinal bleeding from angiodysplasias. This is likely the result of further increase in shear stress and cleavage of HMWM vWF. The study of Grosman-Rimon and colleagues ${ }^{3}$ further expands on that concept, because pulsatility index was negatively correlated with cGMP levels. Platelet-derived growth factor (PDGF) is a critical mediator of angiogenesis, and it is associated with the development of vasoproliferative lesions in several chronic inflammatory conditions. ${ }^{10}$ The contribution of PDGF to angiodysplasia development is unknown. The lower PDGF levels associated with LVADs may be a surrogate for platelet inhibition.

Although many of these changes in coagulation were present in a large proportion of patients with CFLVADs, only a relatively small proportion of them had gastrointestinal bleeding develop. It is therefore very likely that the combination of several of these factors with other, as yet unrecognized, factors is necessary for the occurrence of gastrointestinal bleeding. The results presented in the study of Grosman-Rimon and colleagues $^{3}$ do not allow us to determine which of these mechanism is most prevalent, but they certainly highlight the potential central role of platelets in gastrointestinal bleeding after CF-LVADs.

\section{References}

1. Kirklin JK, Naftel DC, Pagani FD, Kormos RL, Stevenson LW, Blume ED, et al Sixth INTERMACS annual report: a 10,000-patient database. J Heart Lung Transplant. 2014;33:555-64.

2. Boyle AJ, Jorde UP, Sun B, Park SJ, Milano CA, Frazier OH, et al; HeartMate II Clinical Investigators. Pre-operative risk factors of bleeding and stroke during left ventricular assist device support: an analysis of more than 900 HeartMate II outpatients. J Am Coll Cardiol. 2014;63:880-8.

3. Grosman-Rimon L, Tumiati LC, Fuks A, Jacobs I, Lalonde SC, Cherney DZ, et al. Increased levels of cGMP in recipients of continuous flow LVADs: implications for gastrointestinal bleeding. J Thorac Cardiovasc Surg. 2016;151: 219-27.

4. Smolenski A. Novel roles of cAMP/cGMP-dependent signaling in platelets. $J$ Thromb Haemost. 2012;10:167-76.

5. Chen Y, Park S, Li Y, Missov E, Hou M, Han X, et al. Alterations of gene expression in failing myocardium following left ventricular assist device support. Physiol Genomics. 2003;14:251-60.

6. Klovaite J, Gustafsson F, Mortensen SA, Sander K, Nielsen LB. Severely impaired von Willebrand factor-dependent platelet aggregation in patients with a continuous-flow left ventricular assist device (HeartMate II). J Am Coll Cardiol. 2009;53:2162-7.

7. Uriel N, Pak SW, Jorde UP, Jude B, Susen S, Vincentelli A, et al. Acquired von Willebrand syndrome after continuous-flow mechanical device support contributes to a high prevalence of bleeding during long-term support and at the time of transplantation. J Am Coll Cardiol. 2010;56:1207-13.

8. Demirozu ZT, Radovancevic R, Hochman LF, Gregoric ID, Letsou GV, Kar B, et al. Arteriovenous malformation and gastrointestinal bleeding in patients with the HeartMate II left ventricular assist device. J Heart Lung Transplant. 2011;30:849-53

9. Wever-Pinzon O, Selzman CH, Drakos SG, Saidi A, Stoddard GJ, Gilbert EM, et al. Pulsatility and the risk of nonsurgical bleeding in patients supported with the continuous-flow left ventricular assist device HeartMate II. Circ Heart Fail. 2013;6:517-26.

10. Hu W, Huang Y. Targeting the platelet-derived growth factor signaling in cardiovascular disease. Clin Exp Pharmacol Physiol. August 16, 2015 [Epub ahead of print]. 